\title{
Definitive chemoradiotherapy of limited-disease small cell lung cancer: Retrospective analysis of new predictive factors affecting treatment results
}

\author{
TETSUYA KOMATSU, YUKIO OIZUMI, ETSUO KUNIEDA, YOSHIFUMI TAMAI, TAKESHI AKIBA and ASUKA KOGAWA \\ Department of Radiation Oncology, Tokai University School of Medicine, Isehara, Kanagawa 259-1193, Japan
}

Received March 1, 2011; Accepted July 4, 2011

DOI: $10.3892 / \mathrm{ol} .2011 .361$

\begin{abstract}
The aim of this study was to evaluate potential predictive factors in the treatment of limited-disease small cell lung cancer (LD-SCLC). A total of 33 patients with LD-SCLC who underwent definitive chemoradiotherapy at our institute between April 1996 and May 2007 were enrolled in our retrospective study. The relationship between a range of potential predictive factors and the initial response, time to progression and pattern of failure was analyzed. The factors evaluated included the tumor markers Pro-gastrin-releasing peptide (Pro-GRP) and neuron-specific enolase; net tumor size (sum of each lesion mass on computed tomography at 1-cm intervals); total radiation dose; biological effective dose (BED); overall treatment time (OTT); time between the start of any type of treatment and the end of radiation therapy (SER). In addition, the novel factors of radiation dose-intensity $(\mathrm{RDI}=$ $\mathrm{BED} / \mathrm{OTT}$ ) and RDI/NTS (= RDI/net tumor size) were defined. Of the 33 patients evaluated in our study, $22(67 \%)$ achieved a complete response (CR) and $27(82 \%)$ experienced treatment failure or recurrence. High RDI/NTS values showed a significant correlation with $\mathrm{CR}(\mathrm{P}=0.043)$. Prolonged OTT and lower values of RDI and RDI/NTS showed a significant correlation with recurrence within 12 months $(\mathrm{P}=0.022,0.033$ and 0.015 , respectively). The lower values of RDI and RDI/NTS showed a significant correlation with distant metastasis as a first failure site $(\mathrm{P}=0.038$ and 0.044 , respectively). Patients with RDI/NTS $\geq 0.08$ had a more favorable prognosis $(\mathrm{P}=0.045)$. Thus, RDI and RDI/NTS may become beneficial predictive factors in the treatment of LD-SCLC. However, further studies are required to confirm our preliminary results.
\end{abstract}

Correspondence to: Dr Tetsuya Komatsu, Department of Radiation Oncology, Tokai University School of Medicine, 143 Shimokasuya, Isehara, Kanagawa 259-1193, Japan

E-mail: komat@tokai-u.jp

Key words: radiotherapy, small cell lung carcinoma, tumor size, dose-response relationship, overall treatment time

\section{Introduction}

Patients with small cell lung cancer (SCLC) account for approximately $13 \%$ of all lung cancer patients and have a poor prognosis (1). Although $30-40 \%$ of these patients are diagnosed as having limited-disease small cell lung cancer (LD-SCLC), the majority of SCLC patients experience occult metastasis. Systemic chemotherapy is thus considered to be the primary treatment modality. As a consequence, the role of radiotherapy in the treatment of SCLC has been controversial.

However, two meta-analyses carried out in 1992 confirmed the value of thoracic irradiation in decreasing local recurrence and improving survival $(2,3)$. Following these findings, it was shown that concurrent chemoradiotherapy and accelerated hyperfractionation were delivered safely, and 5-year overall survival was improved to $26 \%$ (4). Prophylactic cranial irradiation (PCI) was recommended for patients achieving a complete response (CR). A meta-analysis conducted by Aupérin et al revealed that PCI improved 3-year survival by $5.4 \%$ in patients who achieved CR (5).

Due to these advances in treatment during the previous two decades, certain subgroups of patients with LD-SCLC were expected to achieve a CR. To obtain data regarding curability, a simultaneous analysis of tumor-related and treatment factors was required. Factors such as large tumor size indicate that patient cure may be difficult. It may also be difficult to achieve a cure if the patient has a tumor with a high level tumor marker. In such cases, the possibility of systemic spread exists, even if the cancer has the appearance of localized disease on imaging.

Since treatments for LD-SCLC are associated with considerable toxicity, prediction of patient curability is crucial in determining the appropriate treatment strategy. Patients expected to be cured should be treated with curative intent. Other patients, such as the elderly or poor performance status patients, should be treated with palliative intent.

In the present study, a new approach was used to analyze the relationship between these potential predictive factors and the initial response or pattern of failure, with the objective of gaining new insights into the curability of LD-SCLC.

\section{Patients and methods}

Patients. A total of 45 patients with LD-SCLC, without malignant pleural effusion, excluding patients with follow-up 
Table I. Patient characteristics.

\begin{tabular}{lc}
\hline Characteristic & Value \\
\hline Age (years) & $47-82$ \\
Range & 64 \\
Median & \\
Gender (n) & 29 \\
Male & 4 \\
Female & \\
PS (ECOG) & 19 \\
0 & 11 \\
1 & 3 \\
2 & \\
NSE (ng/ml) & $6.4-120$ \\
Range & 14 \\
Median & \\
Pro-GRP (pg/ml) & $12.3-15,600$ \\
Range & 139 \\
Median & \\
Net tumor size (cm) & $2-93$ \\
Range & 33 \\
Median & \\
\hline
\end{tabular}

PS, performance status; NSE, neuron-specific enolase; Pro-GRP, pro-gastrin-releasing peptide.

periods of less than 6 months, underwent definitive chemoradiotherapy at our institution, between April 1996 and May 2007. Of the 45 patients, 33 were included in our study. Of the 12 patients excluded from the study, 2 were excluded because the primary lesion could not be identified, 8 due to a difficulty in the evaluation of imaging and 2 for having undergone surgery for early-stage primary lesion following completion of radiotherapy. The 33 cases were pathologically confirmed as SCLC and staged using brain magnetic resonance imaging (MRI), computed tomography (CT) of the thorax and upper abdomen, and bone scintigraphy. Bone marrow aspiration was not carried out for any of the patients. Limited stage was defined as the tumor being located in one hemi-thorax, including a contralateral mediastinal node, and the bilateral supraclavicular fossa. Approval for this retrospective analysis was obtained from the Institutional review board. Patient and tumor characteristics are shown in Table I.

Radiotherapy. External-beam radiotherapy was delivered using a linear accelerator with a 6,15 and $18 \mathrm{MV}$ photon beam (6 MV, 11 cases; $15 \mathrm{MV}, 8$ cases and $18 \mathrm{MV}, 14$ cases). Radiation fields typically included the primary lesion, ipsilateral hilum and the entire mediastinum. For 6 patients, the radiation field included only the primary lesion and involved lymph nodes. The supraclavicular fields were treated for 5 patients with supraclavicular fossa nodal involvement. The patients were treated with anterior-posterior opposing fields to a prescribed total radiation dose of 30-45 Gy, followed by irradiation of off-cord oblique opposing fields to a total prescribed dose of 45-60 Gy.
Table II. Timing of chemotherapy and the number of chemotherapy cycles.

\begin{tabular}{lrrrr}
\hline \multicolumn{2}{c}{ Chemotherapy regimen } & & \multicolumn{2}{c}{ No. of chemotherapy cycle } \\
\cline { 1 - 1 } \cline { 5 - 5 } Agents and timing & $\mathrm{n}$ & & Chemotherapy cycle & $\mathrm{n}$ \\
\hline CBDCA+VP-16 & 20 & & $\leq 2$ & 5 \\
Concurrent & 16 & & 3 & 2 \\
Sequential & 4 & & 4 & 6 \\
CDDP+VP-16 & 9 & & 6 & 2 \\
Concurrent & 4 & & & \\
Sequential & 5 & & & \\
CDDP+CPT-11 & 4 & & & \\
Sequential & 4 & &
\end{tabular}

CBDCA, carboplatin; CDDP, cisplatin; VP-16, etoposide; CPT-11, irinotecan hydrochloride hydrate.

Chemotherapy. Table II shows the timing of chemotherapy relative to radiotherapy, the combined agents and the number of chemotherapy cycles. Chemotherapy regimens were selected according to patient age or performance status.

Evaluation and statistical analysis. The factors evaluated were pro-gastrin-releasing peptide (Pro-GRP), neuron-specific enolase (NSE), net tumor size (NTS), total radiation dose, biological effective dose (BED: $\alpha / \beta=10$ ), overall treatment time of radiotherapy (OTT) and time between the start of any treatment and the end of radiation therapy (SER). To compare the effects of different fraction sizes and total doses, the BED was used in a linear-quadratic model. The BED was then calculated as follows, adopting 10 as the $\alpha / \beta$ ratio for the estimation of tumor response: $\mathrm{BED}=\mathrm{nd}(1+\mathrm{d} / \alpha / \beta)$, where $\mathrm{n}=$ fraction number, $\mathrm{d}=$ daily dose and $\mathrm{nd}=$ total dose.

If chemotherapy and radiotherapy were not administered concurrently, tumor marker values prior to the start of the first treatment were used for the analysis. Pro-GRP was not achieved in 2 cases. Net tumor size was defined as the sum of the long axis distance assessed for all of the mass lesions, including the lymph nodes and the primary lesion on each slice of the CT image at $1-\mathrm{cm}$ intervals, using spiral CT prior to planning (Fig. 1). Net tumor size was evaluated prior to commencement of any treatment.

To evaluate these factors synchronously, the following parameters were defined: radiation dose-intensity $(\mathrm{RDI})=$ $\mathrm{BED} / \mathrm{OTT}$; and RDI/NTS = RDI/net tumor size. The relationship between the values of these factors and the three therapy results, i.e., the initial response (CR vs. non-CR), time to progression ( $\leq 12$ vs. $\geq 13$ months) and pattern of failure (loco-regional failure vs. distant metastasis), were analyzed using the Mann-Whitney $\mathrm{U}$ test. $\mathrm{P}<0.05$ was considered to be statistically significant. An initial response was evaluated within 1 month after the completion of radiotherapy and was judged according to RECIST criteria. A CR was defined as the disappearance of all visible disease when assessed using chest X-rays and the normalization of tumor markers. CT was only used to assess the response to treatment when no evaluable 


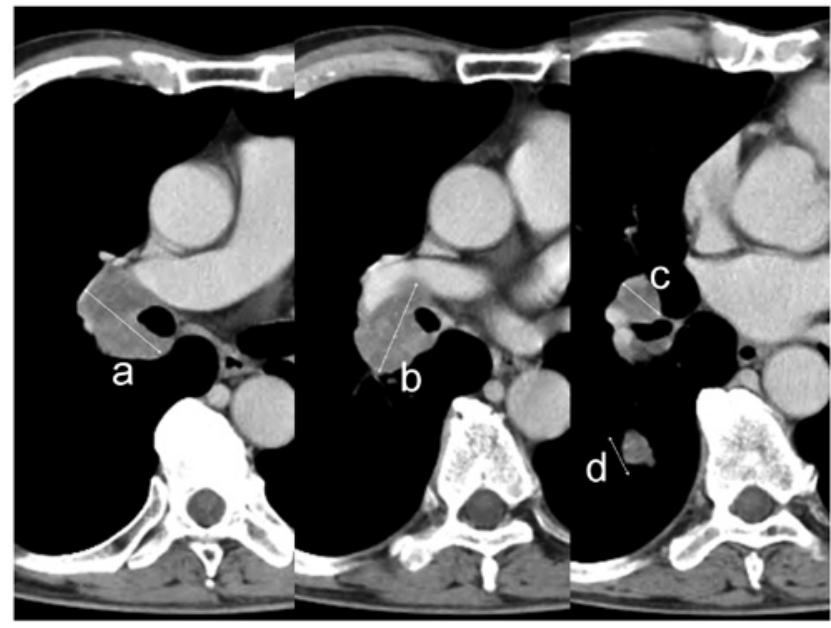

Figure 1. Sample showing the manner in which tumor volume was calculated. Long axis distances were measured for the respective nodules, without distinguishing the primary lesion from lymph node metastasis. Tumor volume was defined as the sum of the long axis distance of each CT slice at a $1-\mathrm{cm}$ interval (i.e., tumor volume $=\mathrm{a}+\mathrm{b}+\mathrm{c}+\mathrm{d}$ ).

lesions were observed on the initial chest X-ray. The time to progression was calculated from the last day of radiotherapy to the date of the first evidence of disease progression. The intervals between subsequent follow-up examination using CT imaging of the chest and abdomen or brain MRI were variable. When the level of any tumor marker became elevated, further examination was performed immediately, in addition to the routine follow-up examination occurring every 1-3 months. If concurrent loco-regional failure and distant metastasis were observed, they were classified as distant metastasis. Using the Kaplan-Meier method, survival time was measured from the date of administration of any initial treatment to the date of death from any cause. The log-rank test was used to evaluate any difference. Statistical analysis was carried out using SPSS version 16.0 software (SPSS Inc., Chicago, IL, USA).

\section{Results}

Tumor progression. The follow-up period ranged from 6 to 80 months, with a median value of 18 months. The 2 -year survival rate was $44 \%$ (95\% CI, 27-61) and the median survival time was 19 months. Of the 33 patients, 22 achieved CR (67\%). Only 4 patients who achieved a CR underwent PCI. In total, 27 patients experienced treatment failure or recurrence. The median time to progression was 6 months. Tumor progression occurred within 12 months in $74 \%$ of the patients.

The pattern of failure and the sites of first recurrence are shown in Table III. A total of 6 patients developed locoregional failure and 21 experienced distant metastasis. The most common site of distant failure was the brain, followed by bone.

Treatment characteristics. A summary of treatment characteristics is shown in Table IV. Once-daily radiotherapy was administered to 15 patients and twice-daily radiation therapy was administered to 18 patients. Radiotherapy was administered twice daily at a dose of $1.5 \mathrm{~Gy} /$ fraction to a total dose of 45 Gy in 17 patients, and 51 Gy in 1 patient. Total doses of the
Table III. Pattern of tumor recurrence.

Site $\mathrm{n}$

Locoregional

In-field recurrence

Marginal recurrence

Out-of-field recurrence

Distant metastasis

Brain

Bone

Liver

Spinal cord

Adrenal

Axillary lymph node

6

Table IV. Treatment characteristics.

\begin{tabular}{lc}
\hline Factors & Value \\
\hline OTT (days) & $18-46$ \\
Range & 29 \\
Median & \\
Fraction schedule (n) & 18 \\
Twice-daily & 15 \\
Once-daily & \\
SER (days) & $18-257$ \\
Range & 58 \\
Median & \\
Total dose (Gy) & $36-60$ \\
Range & 45 \\
Median & \\
BED (Gy) & $47-72$ \\
Range & 52 \\
Median & \\
RDI & $1.51-2.74$ \\
Range & 1.89 \\
Median & \\
RDI/NTS & $0.02-1.31$ \\
Range & 0.0619 \\
Median & \\
\hline OTT, & \\
\hline
\end{tabular}

OTT, overall treatment time; SER, time between commencement of any treatment until the end of radiotherapy; BED, biological effective dose $(\alpha / \beta=10)$; RDI, BED per day; RDI/NTS, RDI/net tumor size.

once-daily fraction schedule ranged from 36 to $60 \mathrm{~Gy}$, mainly at a dose of $2 \mathrm{~Gy} /$ fraction.

Findings of statistical analysis. The results of the statistical analysis are recorded in Table V. The high value of RDI/ NTS showed a significant correlation with CR $(\mathrm{P}=0.043)$. The median RDI/NTS was 0.078 (range 0.020-1.31) in patients who achieved a CR, whereas it was 0.054 (range 0.020-0.08) in those who did not. Net tumor size was of marginal significance $(\mathrm{P}=0.075)$ and patients with larger tumor size tended not to 
Table V. Results of statistical analysis of the experimental date.

\begin{tabular}{lccc}
\hline & \multicolumn{3}{c}{ P-value } \\
\cline { 2 - 4 } Factor & $\begin{array}{c}\text { Initial } \\
\text { response } \\
\text { (CR vs. } \\
\text { non-CR) }\end{array}$ & $\begin{array}{c}\text { Time to } \\
\text { progression } \\
\text { ( } \leq 12 \text { vs. } \geq 13 \\
\text { months })\end{array}$ & $\begin{array}{c}\text { Site of } \\
\text { failure } \\
\text { (LRF vs. } \\
\text { DM) }\end{array}$ \\
\hline Pro-GRP & 0.577 & 0.412 & 0.504 \\
NSE & 0.708 & 0.632 & 0.054 \\
NTS & 0.075 & 0.126 & 0.210 \\
OTT & 0.836 & 0.022 & 0.070 \\
SER & 0.587 & 0.342 & 0.280 \\
BED & 0.762 & 0.061 & 0.114 \\
RT dose & 0.685 & 0.051 & 0.106 \\
RDI & 0.826 & $\mathbf{0 . 0 3 3}$ & $\mathbf{0 . 0 3 8}$ \\
RDI/NTS & $\mathbf{0 . 0 4 3}$ & $\mathbf{0 . 0 1 5}$ & $\mathbf{0 . 0 4 4}$ \\
\hline
\end{tabular}

Pro-GRP, pro-gastrin-releasing peptide; NSE, neuron-specific enolase NTS, net tumor size; OTT, overall treatment time; SER, time between commencement of any treatment until the end of radiotherapy; RT dose, radiation dose; BED, biological effective dose; RDI, BED per day; RDI/NTS, RDI/net tumor size. Bold, statistically significant.

achieve a CR. Median net tumor size was $30 \mathrm{~cm}$ (range 2-93) in patients who achieved CR, whereas it was $37 \mathrm{~cm}$ (range 24-77) in patients who did not.

With regard to the time to progression, prolonged OTT and the lower value of RDI and RDI/NTS showed a significant correlation with disease recurrence within 12 months $(\mathrm{P}=0.022$, 0.033 and 0.015 , respectively). In patients who relapsed within 12 months, the median OTT, RDI and RDI/NTS values were 36 days (range 20-46), 1.79 (range 1.51-2.60) and 0.052 (range 0.02-0.43), respectively, whereas in patients who did not relapse within 12 months or who did not relapse at all, these values were 21 days (range 18-43), 2.47 (range 1.67-2.74) and 0.078 days (range $0.04-1.31$ ), respectively. The BED and total dose exhibited marginal significance when correlated with disease recurrence $(\mathrm{P}=0.061$ and 0.051 , respectively).

Patients who developed a recurrence within 12 months had a higher BED and total radiation dose, which may have been correlated with a longer OTT. The median BED and total dose were $60 \mathrm{~Gy}$ (range 52-72) and $50 \mathrm{~Gy}$ (range 45-60) in patients with recurrence at $\leq 12$ months, respectively, whereas the median BED and total dose were 52 Gy (range 47-72) and 45 Gy (range 36-60) in patients with recurrence within $\geq 13$ months, respectively.

With regard to the site of first failure, the lower value of RDI and RDI/NTS showed a significant correlation with distant metastasis as a first failure site $(\mathrm{P}=0.038$ and 0.044 , respectively). RDI and RDI/NTS were higher in patients who relapsed as a loco-regional failure rather than distant metastasis. The median RDI and RDI/NTS were 1.79 (range 1.51-2.60) and 0.055 (range 0.02-0.43), respectively, in patients who developed distant metastasis as a first failure site, whereas the median RDI and RDI/NTS were 2.41 (range 1.71-2.74) and 0.086 (range $0.05-1.31$ ), respectively, in patients who

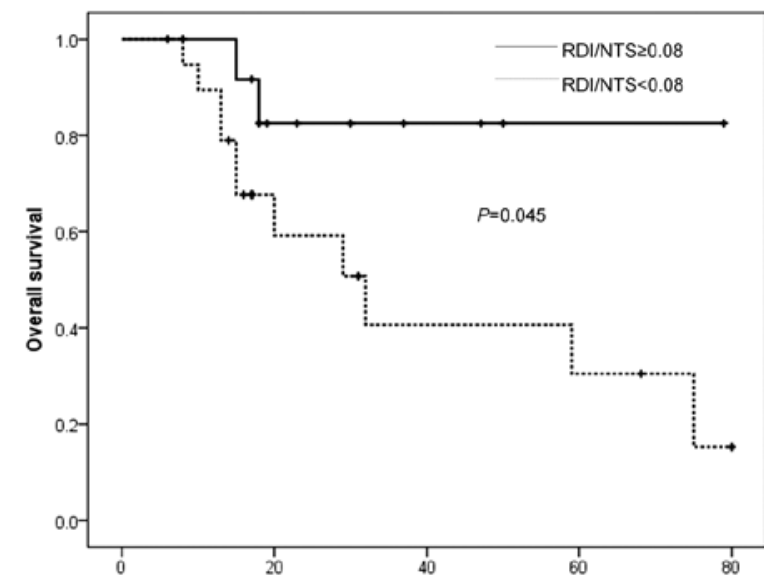

Figure 2. Overall survival curve according to the value of RDI/NTS.

developed loco-regional failure as a first failure site. NSE and OTT exhibited marginal significance $(\mathrm{P}=0.054$ and 0.070 , respectively). In patients who developed distant metastasis, there was a tendency to have a higher NSE value and a longer OTT. In patients with distant metastasis, the median values of NSE and OTT were $17.0 \mathrm{ng} / \mathrm{ml}$ (range 7.9-120.0) and 35 days (range 20-46), respectively, whereas in patients with loco-regional failure, the median values of NSE and OTT were $11.5 \mathrm{ng} / \mathrm{ml}$ (range 7.3-42.0) and 22 days (range 18-42), respectively.

Fig. 2 shows the survival curve according to the value of RDI/NTS. Although the evaluation was limited by a short follow-up period, patients with RDI/NTS $\geq 0.08$ had a more favorable prognosis $(\mathrm{P}=0.045)$.

\section{Discussion}

During the last two decades, the survival of patients with LD-SCLC has improved due to the development of optimal radiotherapy treatment protocols. In these protocols, radiation dose and time factors are crucial. Tumor markers are considered to be associated with outcome (6-10). In addition to these factors, we proposed that tumor size affected treatment outcome. Evaluating these predictive factors is beneficial to the development of effective treatment strategies. For example, treatment of elderly patients using an intensive protocol improves the survival rate, but may simultaneously increase the risk of severe toxicity or treatment-related death $(11,12)$. We hypothesized that patients who are expected to achieve a $\mathrm{CR}$, with no recurrence within 1 year and no progression to systemic disease even if recurrence has occurred, should be treated with curative intent. Therefore, we carried out a retrospective examination of the relationship between these factors and the initial therapeutic response or pattern of failure.

In cases with high tumor marker values, there is a possibility that the disease has already metastasized to other organs. A number of investigators have reported the effectiveness of using tumor markers to evaluate the disease (6-10). The majority of these studies were concerned with NSE or Pro-GRP. Pro-GRP has been shown to have a high sensitivity and to be effective in the diagnosis of cases of early-stage SCLC, or in monitoring the disease after treatment $(7,10)$. On 
the other hand, NSE is often elevated in patients with extended disease, and higher NSE values indicate systemic spread $(7,9,13,14)$. Thus, NSE may be an indicator of systemic spread of the disease. Our study also showed that patients with a high value of NSE exhibited a tendency to develop distant metastasis as an initial relapse site. It is possible that a high value of NSE indicates the existence of distant metastasis, even if it was not detected using imaging.

Tumor size has a great effect on treatment outcome (15). We consider that the reason for the limited studies available regarding tumor size in the case of SCLC is due to the difficulty in measuring tumor size objectively and adequately. Numerous patients with SCLC present with a multiform appearance of the disease due to the fusion of lymph nodes with the primary lesion, rendering assessment of the disease a challenge. Therefore, we measured the length of the long axis distances of each lesion at 1-cm slice intervals on $\mathrm{CT}$, and did not separate the mass into lymph nodes and primary lesion.

Large tumor size is also correlated with poor initial response or local control in patients with LD-SCLC. One treatment strategy for such cases is dose escalation. Choi and Carey stated the need for dose escalation on the basis of a dose-response curve (16). The Cancer and Leukemia Group B (CALGB) 39808 have stated that 70 Gy thoracic radiotherapy delivered concurrently with chemotherapy was feasible and comparable to AHF (17). However, in CALGB 30002, relapse or progression occurred more frequently in the high-dose volume (18). On the other hand, Tomita et al reported the effectiveness of a high dose of radiation (19). Therefore, the benefit of dose escalation remains unclear. We speculate that simple dose escalation cannot improve treatment outcome in cases with large tumors. Evaluation of the true dose-response relationship is difficult. Since dose escalation with a standard fraction schedule causes prolongation of the OTT, which allows additional time for accelerated tumor clonogen proliferation, higher radiation doses are required (20). Recently, a study related to this issue was published (21). Using a mathematical model that took into consideration tumor doubling time and kick-off time, Arvidson et al demonstrated that the most effective duration of the OTT is 3 weeks. These authors reported that if the OTT was fixed at 3 weeks, 2-year local progression-free survival was affected by the dose per fraction, and that dose escalation improved 2-year progression-free survival.

In addition, a recent meta-analysis disclosed that SER was the most important predictive factor when using platinumbased chemotherapy $(22,23)$. A phase III study conducted by Takada et al involving 231 patients with LD-SCLC showed a similar tendency in favor of short SER, but there was no significant difference in survival (24). In this study, brain metastasis occurred more frequently in patients with longer SER, but the loco-regional failure rate was not significantly different. Similarly, Murray et al reported that prolongation of SER resulted in more frequent brain metastasis, although the local control rates were similar (25). We speculate that SER affects survival due to the probability of distant metastasis. However, this tendency was not demonstrated in the present study due to the small number of cases.

Another limitation of the present study was the low rate of PCI. A national survey conducted by Uno et al revealed that PCI has yet to be widely accepted in Japan (26).
Selection bias affected the outcome of this study. However, our analysis indicated the significance of dose escalation without prolongation of the OTT for patients with large tumors. Further studies are required to confirm our preliminary results.

\section{References}

1. Govindan R, Page N, Morgensztern D, Read W, Tierney R, Vlahiotis A, Spitznagel EL and Piccirillo J: Changing epidemiology of small-cell lung cancer in the United States over the last 30 years: analysis of the surveillance, epidemiologic, and end results database. J Clin Oncol 24: 4539-4544, 2006.

2. Pignon JP, Arriagada R, Ihde DC, et al: A meta-analysis of radiotherapy for small-cell lung cancer. N Engl J Med 327: 1618-1624, 1992.

3. Warde $P$ and Payne D: Does thoracic irradiation improve survival and local control in limited-stage small-cell carcinoma of the lung? A meta-analysis. J Clin Oncol 10: 890-895, 1992.

4. Turrisi AT III, Kim K, Blum R, Sause WT, Livingston RB, Komaki R, Wagner H, Aisner S and Johnson DH: Twice-daily compared with once-daily thoracic radiotherapy in limited small-cell lung cancer treated concurrently with cisplatin and etoposide. N Engl J Med 340: 265-271, 1999.

5. Aupérin A, Arriagada R, Pignon JP, le Péchoux C, Gregor A, Stephens RJ, Kristjansen PE, Johnson BE, Ueoka H, Wagner H and Aisner J: Prophylactic cranial irradiation for patients with small-cell lung cancer in complete remission. N Engl J Med 341: 476-484, 1999 .

6. Yonemori K, Sumi M, Fujimoto N, Ito Y, Imai A, Kagami Y and Ikeda $\mathrm{H}$ : Pro-gastrin-releasing peptide as a factor predicting the incidence of brain metastasis in patients with small cell lung carcinoma with limited disease. Cancer 104: 811-816, 2005.

7. Shibayama T, Ueoka H, Nishii K, Kiura K, Tabata M, Miyatake K, Kitajima $\mathrm{T}$ and Harada M: Complementary roles of pro-gastinreleasing peptide (ProGRP) and neuron specific enolase (NSE) in diagnosis and prognosis of small-cell lung cancer (SCLC). Lung Cancer 32: 61-69, 2001.

8. Fizazi K, Cojean I, Pignon JP, Rixe O, Gatineau M, Hadef S, Arriagada R, Baldeyrou P, Comoy E and le Chevalier T: Normal serum neuron specific enolase (NSE) value after the first cycle of chemotherapy. Cancer 82: 1049-1055, 1998

9. Johnson PW, Joel SP, Love S, Butcher M, Pandian MR, Squires L, Wrigley PF and Slevin ML: Tumor markers for prediction of survival and monitoring of remission in small cell lung cancer. Br J Cancer 67: 760-766, 1993.

10. Sunaga N, Tsuchiya S, Minato K, Watanabe S, Fueki N, Hoshino H, Makimoto T, Ishihara S, Saito R and Mori M: Serum pro-gastrin-releasing peptide is a useful marker for treatment monitoring and survival in small-cell lung cancer. Oncology 57: 143-148, 1999.

11. Findlay MP, Griffin AM, Raghavan D, McDonald KE, Coates AS, Duval PJ and Gianoutsos P: Retrospective review of chemotherapy for small cell lung cancer in the elderly: does the end justify the means? Eur J Cancer 27: 1597-1601, 1991.

12. Safont MJ, Artal-Cortes A, Sirera R, Gómez-Codina J, González-Larriba JL, Barneto I, Carrato A, Isla D, Rosell R and Camps C: Retrospective study of efficacy and toxicity on patients older than 70 years within a randomized clinical trial of two cisplatin-based combinations in patients with small-cell lung cancer. Lung Cancer 63: 83-87, 2009.

13. Carney DN, Marangos PJ, Ihde DC, Bunn PA Jr, Cohen MH, Minna JD and Gazdar AF: Serum neuron-specific enolase: a marker for disease extent and response to therapy of small-cell lung cancer. Lancet 1: 583-585, 1982.

14. Burghuber OC, Worofka B, Schernthaner G, Vetter N, Neumann M, Dudczak R and Kuzmits R: Serum neuron-specific enolase is a useful tumor marker for small cell lung cancer. Cancer 65: 1386-1390, 1990.

15. Dubben HH, Thames HD and Beck-Bornholdt HP: Tumor volume: a basic and specific response predictor in radiotherapy. Radiother Oncol 47: 167-174, 1998.

16. Choi NC and Carey RW: Importance of radiation dose in achieving improved loco-regional tumor control in limited stage small-cell lung carcinoma: an update. Int J Radiat Oncol Biol Phys 17: 307-310, 1989. 
17. Bogart JA, Herndon JE II, Lyss AP, Watson D, Miller AA, Lee ME, Turrisi AT and Green MR: 70 Gy thoracic radiotherapy is feasible concurrent with chemotherapy for limited-stage small-cell lung cancer: analysis of Cancer and Leukemia Group B study 39808. Int J Radiat Oncol Biol Phys 59: 460-468, 2004.

18. Miller AA, Wang XF, Bogart JA, Hodgson LD, Rocha Lima CM, Radford JE, Vokes EE and Green MR: Phase II trial of paclitaxeltopotecan-etoposide followed by consolidation chemotherapy for limited-stage small cell lung cancer: CALGB 30002. J Thorac Oncol 2: 645-651, 2007.

19. Tomita N, Kodaira T, Hida T, Tachibana H, Nakamura T, Nakahara R and Inokuchi H: The impact of radiation dose and fractionation on outcomes for limited-stage small-cell lung cancer. Int J Radiat Oncol Biol Phys 76: 1121-1126, 2010.

20. Bentzen SM and Thames HD: Clinical evidence for tumor clonogen regeneration: interpretations of the date. Radiother Oncol 22: 161-166, 1991.

21. Arvidson NB, Khuntia D and Tome WA: Dose escalation model for limited-stage small-cell lung cancer. Radiother Oncol 91: 379-385, 2009.

22. De Ruysscher D, Pijls-Johannesma M, Bentzen SM, et al: Time between the first day of chemotherapy and the last day of chest radiation is the most important predictor of survival in limiteddisease small-cell lung cancer. J Clin Oncol 24: 1057-1063, 2006.
23. Pijls-Johannesma M, de Ruysscher D, Vansteenkiste J, Kester A, Rutten I and Lambin P: Timing of chest radiotherapy in patients with limited stage small cell lung cancer: a systematic review and meta-analysis of randomized controlled trials. Cancer Treat Rev 33: 461-473, 2007.

24. Takada M, Fukuoka M, Kawahara M, Sugiura T, Yokoyama A, Yokota S, Nishiwaki Y, Watanabe K, Noda K, Tamura T, Fukuda H and Saijo N: Phase III study of concurrent vs. sequential thoracic radiotherapy in combination with cisplatin and etoposide for limited-stage small-cell lung cancer: results of the Japan Clinical Oncology Group Study 9104. J Clin Oncol 20: 3054-3060, 2002.

25. Murray N, Coy P, Pater JL, et al: Importance of timing for thoracic irradiation in the combined modality treatment of limited-stage small-cell lung cancer. J Clin Oncol 11: 336-344, 1993.

26. Uno T, Sumi M, Ishihara $Y$, Mitsumori $M$ and Teshima $T$ : Changes in pattern of care for limited-stage small-cell lung cancer: result of the 99-01 pattern of care study. A nationwide survey in Japan. Int J Radiat Oncol Biol Phys 71: 414-419, 2008. 